\title{
Development and Use of a GPS Ionospheric Scintillation Monitor
}

\author{
Theodore L. Beach, Member, IEEE, and Paul M. Kintner, Senior Member, IEEE
}

\begin{abstract}
Besides their intended use in radionavigation, global positioning system (GPS) satellite signals provide convenient radio beacons for ionospheric studies. Among other propagation phenomena, the ionosphere affects GPS signal propagation through amplitude scintillations that develop after radio waves propagate through ionospheric electron density irregularities. This paper outlines the design, testing, and operation of a specialized GPS receiver to monitor L-band amplitude scintillations: the Cornell scintillation monitor. The Cornell scintillation monitor consists of a commercial GPS receiver development kit with its software modified to $\log$ signal strength from up to 12 channels at a high data rate (50 samples/s). Other features of the receiver include the optional assignment of a channel to monitor the receiver noise level in the absence of signal tracking and the means to synchronize measurements between nearby independent receivers to perform drift measurements and correlation studies. The Cornell scintillation monitor provides characterization of the operational L-band scintillation environment and additionally permits study of the multipath environment of a static antenna. GPS scintillation monitors can provide information about the state of ionospheric irregularities for pure research purposes as well. Here their strength lies in the fact that they are inexpensive and compact and therefore can be readily proliferated. Even a single scintillation monitor can supplement radar spatial coverage of irregularities in a limited way because it monitors several satellite lines of sight simultaneously. This article introduces some of the potential of the scintillation monitor for research, primarily through examples associated with field testing the instrument.
\end{abstract}

Index Terms-Equatorial ionosphere, global positioning system (GPS), GPS receivers, ionospheric scintillation, space weather, UHF propagation, UHF scattering.

\section{INTRODUCTION}

$\mathbf{T}$ HE DECLARATION of operational status for the global positioning system (GPS) in 1995 marked a highlight in the history of navigation. No longer constrained by proximity to operating beacons, users can navigate with positioning accuracy of the order of $100 \mathrm{~m}$ (better with selective availability now turned off) worldwide through the standard positioning service and much more accurately with differential aiding. The impact of the ionosphere on the propagation speed of ranging signals was considered early in the development of the GPS signal structure and led to the current dual-frequency system,

Manuscript received May 17, 2000; revised August 28, 2000. This work was supported in part by the Office of Naval Research under Grant N00014-92-J1822, and the Air Force Research Laboratory under AFOSR Task 2311AS.

T. L. Beach is with the Space Vehicles Directorate, Air Force Research Laboratory, Hanscom Air Force Base, MA 01731 USA (e-mail: Theodore. Beach@hanscom.af.mil).

P. M. Kintner is with the School of Electrical Engineering, Cornell University, Ithaca, NY 14853 USA (e-mail: paul@ece.cornell.edu).

Publisher Item Identifier S 0196-2892(01)02148-9. with civilian groups calling for even more frequencies to reduce ranging errors. Nevertheless, one aspect of ionospheric propagation not compensated for in the GPS signal strategy was scintillation from ionospheric irregularities. As GPS receivers become widely used, and as they are integrated into other systems, the potential impact of ionospheric irregularities on these systems grows.

Free electrons in the ionosphere alter its refractive index from that of free space. The greater the total electron content (TEC), the integral of electron density along the line of sight to a satellite, the more phase advance and group delay the GPS signal experiences. Phase advance and group delay affect navigation directly via errors in carrier phase and ranging measurements. Furthermore, if the ionosphere is not smooth but contains electron density irregularities, the irregular phase front of the GPS signal as it exits the ionosphere gives rise to phase and amplitude scintillations on the ground [1]. Severe scintillations of the order of $20 \mathrm{~dB}$ can cause loss of signal for one or more satellites, thus potentially degrading the navigation solution.

Ionospheric irregularities that produce scintillations at GPS frequencies (L band) occur mainly in regions near the geomagnetic poles and equator [2], [3]. The strongest L-band scintillations, with signal fades of $20 \mathrm{~dB}$ or more during solar maximum, occur around $\pm 15^{\circ}$ dip latitude (i.e., in the equatorial anomaly regions) after sunset [3]. Equatorial L-band scintillations usually relate to the phenomenon of equatorial spread $F$ (ESF), while many different phenomena generate scintillations at higher latitudes. In either case, scintillation levels often show extreme variability from day to day in addition to well known seasonal and solar cycle dependencies.

The impact of scintillations on GPS navigation has received renewed attention recently. Several factors account for the current interest. The system has only been officially operational since 1995, so far fewer GPS receivers and only a partial GPS satellite constellation existed during the last solar maximum (1989-1990). Also, increasing reliance on GPS in critical applications such as air traffic control and precision landings is expected. Consequently, concern has grown over the potential impact of scintillations on GPS and the systems that depend on it during the presentsolar maximum in 2000-2001.

To investigate the scintillation environment at GPS frequencies, the Space Physics group at Cornell University, Ithaca, NY, developed a specialized receiver, hereafter termed the Cornell scintillation monitor. The Cornell scintillation monitor is a modification of a commercial GPS development system, the Plessey GPS Builder-2 [4]. The Plessey development system consists of a 16-bit PC card containing RF and correlator chips with accompanying receiver source code written in $C$ and assembly 
language. All receiver functions above the level of code correlation, including code and carrier tracking, devolve upon the PC.

The advantage of using the Plessey development system is that the code correlator outputs are not buried within an onboard microcontroller as is the case in most commercially available GPS receivers. Averaging the "raw" correlations provides a known, albeit uncalibrated, measurement of signal strength. The Cornell scintillation monitor outputs signal strength data at 50 samples/s for each satellite tracked, with data collection synchronized to the data bits of the GPS navigation message. The scintillation monitor also provides phase data at 10 samples/s, although finding phase scintillations with the current receiver design, amid oscillator and tracking loop noise and large background trends, has proven problematic. For the most part, then, we focus on amplitude measurements.

Another group has also developed a GPS ionospheric scintillation monitor recently, under contract to the U.S. Air Force [5], [6]. The approaches of the two projects are somewhat different, however. The Air Force receiver, also known as the ionospheric scintillation monitor (ISM), is normally intended to operate as part of a remote, self-contained scintillation alert system. It mostly reports signal statistics, although raw data are optionally available (for a limited number of channels). The Cornell scintillation monitor makes detailed recordings of all available data (up to 12 channels) for high time resolution studies of amplitude scintillations. Signal statistics are available only after postprocessing. The ISM also has the option of a higher quality oscillator than the Cornell scintillation monitor has, and its developers claim more success in taking phase measurements.

Nevertheless, each GPS scintillation monitor has its niche. The ISM is better suited to studies of scintillation parameters and to unattended, long-term operation. The Cornell receiver is better suited to detailed examination of scintillation spectral and temporal characteristics. The latter receiver is also better utilized in campaign-style operation because of the larger quantity of data recorded ( $~ 200 \mathrm{MB}$ per 12-h period, depending on the number of satellites visible) and the need for operator intervention every few days to back up the data. The availability of raw data for all channels makes cross-correlation studies (as in [7]) feasible with the Cornell scintillation monitor. Finally, the $50-\mathrm{Hz}$ sampling rate permits investigation of a larger range of the fluctuation spectrum than in typical GPS receivers.

The key advantages of GPS scintillation monitors for research purposes are their low cost and small size. Although their ionospheric data return is by no means as rich as that of incoherent scatter radar installations, for example, scintillation monitors can be readily proliferated to monitor ionospheric irregularities with broad spatial coverage. Furthermore, with increasing use of scintillation monitors, we are optimistic that novel techniques will be developed to eke out the last bit of ionospheric information that they can provide, probably in combination with other available ionospheric instrumentation.

This paper outlines the development, testing, and use of the Cornell scintillation monitor. Testing includes bench testing with a single-channel GPS simulator and field measurements from an equatorial spread $F$ campaign in South America near the magnetic equator. The discussion of field testing includes the important topic of distinguishing amplitude scintillation from other sources of signal strength variance, particularly multipath.

\section{GPS RECEIVER BACKGROUND}

This section describes features of GPS that make receiving its signals different from conventional satellite beacon techniques that are traditionally used in scintillation work. Using this background, Section III describes in some detail the signal strength measurements that are required to monitor amplitude scintillations on GPS signals. These two sections provide the basic background for making signal strength measurements using GPS and for applying them to scintillation measurements. This information exists to a certain extent in the GPS literature, but not always in an accessible form.

A GPS relies on code division multiple access (CDMA) spread-spectrum techniques to provide precise ranging measurements and to allow navigation signals from multiple satellites to share the same spectrum allocation. Because GPS is a CDMA system, acquiring and tracking GPS signals involves two degrees of freedom in the receiver: code offset (the time offset between the broadcast code and the receiver's code) and frequency. In contrast, traditional scintillation receivers use beacon signals and need only "tune in" the carrier frequency, compensating for Doppler shift and oscillator drift.

To sense the code offset, the receiver monitors the cross-correlation between the received pseudo-random noise (PRN) code unique to each satellite and a locally generated replica. In the case of the civil GPS signal, the PRN code, called the coarse/acquisition (C/A) code, has a 1.023-MHz chipping rate and a repetition period of $1 \mathrm{~ms}$ (a"chip" is the length of one bit of the PRN code and the "chipping rate" is the PRN code rate, as opposed to the lower data bit rate defined below). The specific PRN code used, out of a set of 32 assigned codes [8], uniquely identifies each satellite. Common practice refers to the various satellites as PRN $x$, where $x$ is $1-32$.

The appropriate C/A PRN binary code $( \pm 1)$ modulates the $1.57542 \mathrm{MHz}$ (L1) carrier of each satellite at the chipping rate. At longer time scales, the data bits of the GPS navigation message $( \pm 1)$ modulate the broadcast signal at a $50-\mathrm{Hz}$ rate. Each data bit covers $20 \mathrm{~ms}$ or $20 \mathrm{C} / \mathrm{A}$ code periods. Thus, the civil broadcast signal $S(t)$ is essentially in the following form:

$$
S(t)=B D(t) C(t) \cos \left(\omega_{1} t\right)
$$

where $B$ is the broadcast signal amplitude, $D(t)( \pm 1)$ represents the 50-bit/s data, $C(t)( \pm 1)$ represents the 1.023-MHz C/A code sequence, and $\omega_{1}=2 \pi(1.57542 \mathrm{GHz})$. Fig. 1 depicts the time scales represented by (1) schematically.

Fig. 2 shows a simplified block diagram of the Plessey development system receiver. The powered antenna receives the RF signal, which then passes through four stages of down-conversion in the RF front end (GP2010 chip). At the last stage of down-conversion within the RF front end, the signal is oversampled and converted to a two-bit digital representation (in which values of \pm 1 and \pm 3 are encoded). The front-end chip applies automatic gain control (AGC) to keep the digital representation equal to \pm 1 for $70 \%$ of the time [4]. 


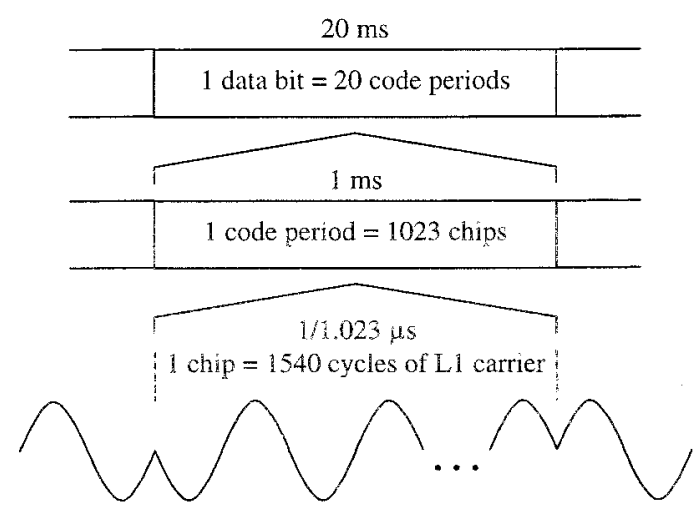

Fig. 1. Schematic representation of GPS C/A code signal structure arranged from (bottom) shortest time scale to (top) longest time scale. Additional structure at longer time scales exists in the data bits to form the GPS navigation message (not shown).

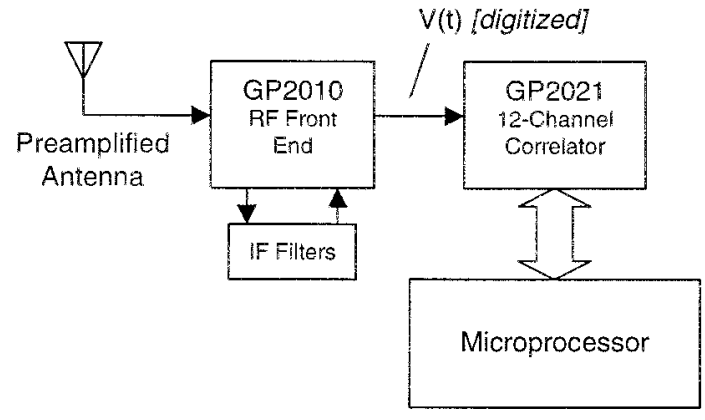

Fig. 2. Block diagram of commercial, single-frequency, open-architecture GPS receiver. The IF filters include an external surface acoustic wave (SAW) module and external LC networks. For the system used in the Cornell scintillation monitor, the controlling microprocessor is a PC.

At the output of the front-end chip, the down-converted signal is predominantly noise-like in its characteristics, consisting of GPS PRN modulation, thermal noise, and any in-band interference. Now the digitized signal passes into the correlator chip (GP2021) for detection. The correlator chip contains 12 identical correlator channels. Each channel may be independently programmed by means of registers under software control with regard to PRN code used, chipping rate, local oscillator frequency, etc. Under normal operation, the baseline Plessey software assigns one channel to each GPS satellite in view (up to $12)$. The software reads the in-phase $(I)$ and quadrature $(Q)$ outputs of the correlator channels every code period by means of an interrupt-driven polling scheme. The correlator outputs provide feedback to the code and carrier frequency tracking loops, which are implemented in software, and to higher level functions like GPS navigation message retrieval and navigation itself.

Fig. 3 shows a simplified block diagram of a single integrate-and-dump channel in the correlator chip. The signal $V(t)$ represents the GPS signal after digitization within the RF front end and has a center frequency of $\sim 1.4 \mathrm{MHz}$. Treating the output of the front-end chip and subsequent signals as analog signals for the purpose of presentation

$$
V(t)=A D C(t+\tau) \cos \left(\omega_{D} t-\phi\right)+n(t)
$$

where $A$ is the received signal amplitude (slowly varying, uncalibrated, and influenced by AGC), and $D$ is the navigation

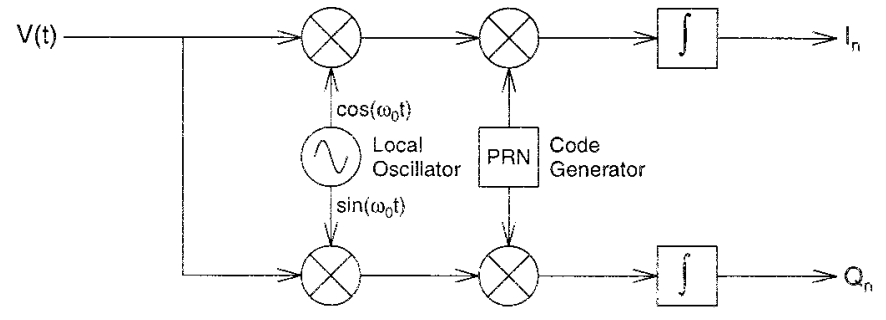

Fig. 3. Simplified block diagram of a single channel correlator module in a GPS receiver. The signal $V(t)$ results from several steps of down conversion of the original RF signal. Additional circuitry (not shown) resets the integrators each C/A code period $(1 \mathrm{~ms})$ and latches the $I_{n}$ and $Q_{n}$ samples.

message data bit (assumed to remain constant over the integration period). As before, $C(\cdots)$ represents the $\mathrm{C} / \mathrm{A}$ code value. Now, though, the signal has a code offset $(\tau)$ and a phase offset $(\varphi)$ relative to the channel's code generator and local oscillator (of frequency $\omega_{0}$ ), respectively. Finally, $\omega_{D}$ is the down-converted signal frequency, and $n(t)$ represents thermal noise. For more discussion of the analysis of strictly digital signals in GPS receivers, we refer the reader to references [9] and [10].

The unmodified Plessey receiver uses a noncoherent delay-lock loop architecture [4], with its implementation split between hardware and software [11], to track the GPS signals once acquired. As delay-lock loops, the tracking loops seek to drive $\tau$ to zero and $\omega_{0}$ to $\omega_{D}$ (but not $\varphi$ to zero). Actually, the correlator chip contains an additional in-phase and quadrature, integrate-and-dump correlator section for each channel. The same code generator drives the additional sections but with a 1/2-chip $(0.489 \mu \mathrm{s})$ delay. With the additional correlator sections, the delay-lock loop's scheme is to drive the early-late correlator power difference to zero [4], [9], with the result that the channel's code generator tracks $1 / 4$ chip ahead of the received code under code lock conditions. In this case, barring severe multipath distortion of the correlation function shape, we recover the $I_{n}$ samples by summing the early and late correlator in-phase outputs (likewise with $Q_{n}$ ).

\section{Power Measurements}

The following formulas give the correlator output samples $I_{n}$ and $Q_{n}$ for the stated form of $V(t)$ :

$$
\begin{aligned}
I_{n} & =\frac{A D T_{c}}{2} R(\tau) \operatorname{sinc}\left(\Delta \omega T_{c} / 2\right) \cos (\phi)+x_{n} \\
Q_{n} & =\frac{A D T_{c}}{2} R(\tau) \operatorname{sinc}\left(\Delta \omega T_{c} / 2\right) \sin (\phi)+y_{n}
\end{aligned}
$$

where $\operatorname{sinc}(u)=\sin (u) / u, \Delta \omega=\omega_{D}-\omega_{0}$, and $R(\tau)$ is the C/A code correlation function

$$
R(\tau) \approx \begin{cases}1-|\tau| / T_{\text {chip }}, & \text { if }|\tau|<T_{\text {chip }}, \\ 0, & \text { otherwise }\end{cases}
$$

letting $T_{\text {chip }}=1 / 1.023 \mu \mathrm{s}$, the length of a C/A code chip. Also, $T_{c}=1 \mathrm{~ms}$ is the C/A code period. The $x_{n}$ and $y_{n}$ are independent, zero-mean, Gaussian random variables with white-noise spectra. These noise terms each have a variance of $N_{0} T_{c} / 4$, where $N_{0}$ is the one-sided spectral density of the noise in the channel in $\mathrm{W} / \mathrm{Hz}$. 
The reader should note that the definition of the amplitude scaling in the $I$ and $Q$ samples represented by (3) and (4) is somewhat arbitrary throughout the literature [9]-[11]. For example, reference [11] chooses to normalize the coefficients of the correlation functions to $D$ and to modify the variance of the noise samples accordingly. Likewise, reference [9] normalizes the noise samples to unit variance and modifies the coefficients of the correlation functions appropriately. Reference [10] uses yet another normalization scheme, also different from our own. We have chosen to retain the $A D T_{c} / 2$ coefficients for clarity of presentation since they are the direct result of the mixing and integrating process (where the factor of $1 / 2$ results from retaining the difference frequency term in the modulation products and discarding the sum frequency term). The $I$ and $Q$ samples in (3) and (4) are proportional to the other results [9]-[11], and the choice of scaling does not significantly affect subsequent analysis.

If the delay-lock loop is tracking, $\tau \rightarrow 0$ (code lock condition) and $\Delta \omega \rightarrow 0$ (frequency lock condition). Therefore, $R(\tau) \rightarrow$ 1 , and $\operatorname{sinc}\left(\Delta \omega T_{c} / 2\right) \rightarrow 1$. Momentarily ignoring the noise terms, then, $I_{n}^{2}+Q_{n}^{2}=A^{2} T_{c}^{2} / 4$ (recalling that $D^{2}$ is identically 1 ). This quantity $I_{n}^{2}+Q_{n}^{2}$ is clearly proportional to signal strength $S=A^{2} / 2$. So the most straightforward estimator of signal strength is an average of $I_{n}^{2}+Q_{n}^{2}$ over several code periods. References [5] and [9] define the following signal strength estimator ("wide band power")

$$
\mathrm{WBP}_{k}=\left[\sum_{n=1}^{M}\left(I_{n}^{2}+Q_{n}^{2}\right)\right]_{k} .
$$

Here $M=20$ is chosen to be the number of C/A code periods in a GPS navigation message data bit, and $k$ represents the data bit number.

The expected value $\left(\mu_{k}\right)$ and variance $\left(\sigma_{k}^{2}\right)$ of the estimator (6) are straightforward to compute using standard methods of calculating signal statistics [12] by exploiting the fact that the noise samples are zero-mean with known variances and uncorrelated. For example, under code lock $(\tau \rightarrow 0)$ and frequency $\operatorname{lock}(\Delta \omega \rightarrow 0)$

$$
\begin{aligned}
\mu_{k} & =E\left\{\mathrm{WBP}_{k}\right\}=\left[\sum_{n=1}^{M} E\left\{I_{n}^{2}\right\}+\sum_{n=1}^{M} \mathrm{E}\left\{Q_{n}^{2}\right\}\right]_{k} \\
& =\frac{M N_{0} T_{c}}{2}\left[1+T_{c}\left(S / N_{0}\right)\right]
\end{aligned}
$$

where $E\{\cdots\}$ is the expected value operator. The quantity $S / N_{0}$ is the signal to noise density ratio (in $\mathrm{Hz}$, since $S$ has units of $W$ and $N_{0}$ is in $\mathrm{W} / \mathrm{Hz}$ ) and is commonly expressed as $C / N_{0}=10 \log _{10}\left(S / N_{0}\right)$, the so-called "carrier-to-noise density in dB-Hz" [9,p. 365]. Similarly, straightforward but tedious algebra shows that, under code and frequency lock conditions

$$
\begin{aligned}
\sigma_{k}^{2} & =E\left\{\left(\mathrm{WBP}_{k}-\mu_{k}\right)^{2}\right\}=E\left\{\mathrm{WBP}_{k}^{2}\right\}-\mu_{k}^{2} \\
& =\frac{M N_{0}^{2} T_{c}^{2}}{4}\left[1+2 T_{c}\left(S / N_{0}\right)\right] .
\end{aligned}
$$

Equations (7) and (8) may be verified against (110) and (112), respectively, of reference [9] by properly accounting for the scaling factor between the current set of $I$ and $Q$ samples and those defined in the reference.

For examining fluctuations in $\mathrm{WBP}_{k}$ on a dB plot, the normalized root mean square (RMS) deviation of $\mathrm{WBP}_{k}$ is handy

$$
\delta_{k}=\frac{\sigma_{k}}{\mu_{k}}=\frac{1}{\sqrt{M}} \frac{\sqrt{1+2 T_{c}\left(S / N_{0}\right)}}{1+T_{c}\left(S / N_{0}\right)} .
$$

If $C / N_{0}=35 \mathrm{db}-\mathrm{Hz}, \delta_{k}=0.14$ yielding about $\pm 0.6 \mathrm{~dB}$ deviation in $\mathrm{WBP}_{k}$ due to noise, for example. Although not immediately evident from the functional form of (9), $\delta_{k}$ increases with decreasing carrier to noise ratio as one would expect.

Another useful signal strength estimator, for summary plot purposes, is the average wide band power over $1 \mathrm{~s}$ intervals

$$
\mathrm{WBP}_{1 \mathrm{~s}}=\frac{1}{50} \sum_{k=1}^{50} \mathrm{WBP}_{k}
$$

Naturally, the normalized RMS deviation of $\mathrm{WBP}_{1 \mathrm{~s}}$ improves to $\sqrt{20 / 1000}=0.14$ of the deviation of the 20 -ms sums because of the longer averaging period. As usual, the tradeoff between time resolution and measurement scatter must be considered carefully before adopting one particular averaging interval. Nonetheless, $\mathrm{WBP}_{1 \mathrm{~s}}$ usually is sufficient to resolve weak equatorial L-band amplitude scintillations.

Other power estimators exist, e.g., "narrow band power" [5], [9]

$$
\operatorname{NBP}_{k}=\left(\sum_{n=1}^{M} I_{n}\right)_{k}^{2}+\left(\sum_{n=1}^{M} Q_{n}\right)_{k}^{2}
$$

Narrow band power, like $\mathrm{WBP}_{k}$, covers 20-ms data bit intervals but exhibits larger measurement scatter (i.e., variance). Also, narrow band power is sensitive to data-bit sign transitions and therefore cannot be readily extended beyond a 20-ms period. Still, for flexibility, the Cornell scintillation monitor logs both wide band power and narrow band power for each data bit period, as determined by the timing of the GPS signal that is received on that channel. We compute $1 \mathrm{~s}$ power averages $\mathrm{WBP}_{1 \mathrm{~s}}$ in post-processing.

As it stands, $\mathrm{WBP}_{k}$ is an uncalibrated measure. The preamplified antennas and analog front ends of the Plessey receiver cards have unknown gains due to factors like manufacturing variations, ambient temperature changes, and component aging. The antenna patterns are not measured or controlled. Even if all of these factors were eliminated or compensated for, the front end would still have an automatic gain control (AGC).

The AGC responds (with a time constant of about $2 \mathrm{~ms}$ [4]) to the average signal level at the point of analog to digital conversion, the point where the $1.4 \mathrm{MHz}$ down-converted signal $V(t)$ is fed into all channels in parallel, and adjusts the IF gain. Thus, intermittent RF interference within the receiver bandwidth or changes in the system noise temperature can cause gain changes. Fortunately, the primary quantities of interest in the study of amplitude scintillation relate to fractional changes in signal strength and not their absolute value (see, 
e.g., $S_{4}$ index below). Unlike a conventional beacon receiver, the scintillations themselves do not cause an AGC response (owing to the spread spectrum nature of the signal).

$\mathrm{WBP}_{k}$ also includes noise power in its expected value, masking some of the percentage fluctuation of true signal strength. In order to monitor the average noise power, and to compensate for some gain changes, the Cornell scintillation monitor provides a "noise channel." The noise channel correlates the incoming signal with a PRN code that no currently orbiting satellite uses. The statistics of $\mathrm{WBP}_{k}$ on the noise channel are ideally the same as those obtained above with the signal strength $S$ set to zero. In practice, we have observed only small, slow variations in the average noise channel $\mathrm{WBP}_{k}$.

These noise channel measurements are important for calculating accurate values of the standard $S_{4}$ scintillation index [1], the normalized RMS deviation of signal strength $S$

$$
S_{4}^{2}=\frac{\left\langle S^{2}\right\rangle-\langle S\rangle^{2}}{\langle S\rangle^{2}}
$$

where, in practice, the angle brackets $\langle\cdots\rangle$ indicate time averages of received signal strength. The computation of $S_{4}$ should ideally include only the effects of ionospheric irregularities on radio wave propagation and not measurement scatter due to noise. Additionally, the noise power should not be permitted to bias the average signal strength upward. Subtracting the noise channel measurement mitigates the latter (we use the autocorrelation function of $\mathrm{WBP}_{k}$ with time to help remove the variance due to measurement scatter).

\section{RECEIVER TESTING}

We conducted two types of testing of the Cornell scintillation monitor, bench testing and field-testing. Bench testing demonstrated, both qualitatively and quantitatively, that the receiver would respond to signal strength fluctuations at rates up to the $25-\mathrm{Hz}$ Nyquist frequency. Field-testing showed that the scintillation monitor responds to equatorial spread $F$ irregularities, as observed by other instruments. Field-testing also verified that the approach used to synchronize data collection between nearby receivers for cross-correlation studies of ionospheric drift is functional. In the context of this paper, the fieldtesting examples additionally provide the means to introduce some of the research potential of the Cornell scintillation monitor.

\section{A. Bench Testing}

Bench testing employed a Northern Telecom STR2775 single-channel GPS simulator. The simulator generates the C/A code modulation for a single, simulated satellite on L1. Signal simulation also includes a dummy navigation message. The GPS simulator resides on a 16-bit PC card and the output signal strength and simulated Doppler shift fall under real-time PC software control. Signal strength is programmable over a \pm 20 $\mathrm{dB}$ range in $0.1-\mathrm{dB}$ increments, while the range of simulated Doppler shifts corresponds to $\pm 15000 \mathrm{~m} / \mathrm{s}$ in line of sight velocity.
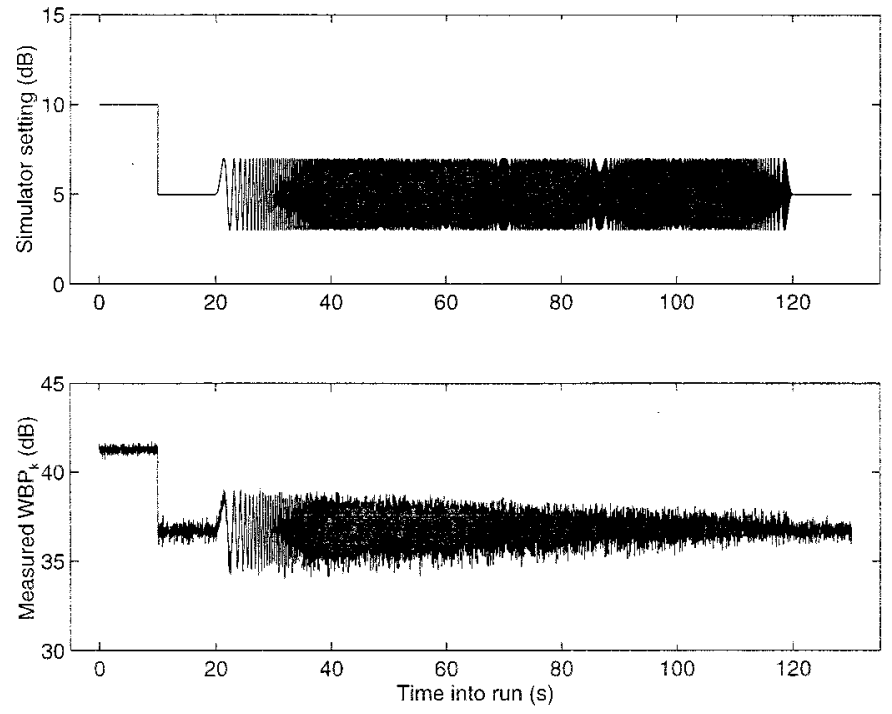

Fig 4 Swept frequency or "chirp" test of scintillation monitor. (Top) Amplitude setting on the simulator as a function of time. The chirp begins $20 \mathrm{~s}$ into the run and lasts $100 \mathrm{~s}$. (Bottom) Scintillation monitor response. The peculiar "envelopes" in the top panel (for example near $70 \mathrm{~s}$ and $85 \mathrm{~s}$ into the run) result from sampling effects.

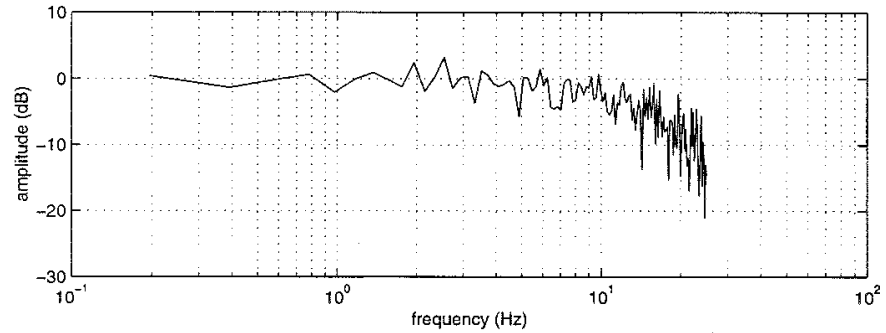

Fig. 5. Amplitude of transfer function between simulator output signal and scintillation monitor response for the chirp test.

Fig. 4 illustrates the results of one particular bench test, the "chirp test," designed to investigate the frequency response of the Cornell scintillation monitor. The top panel shows the software settings of the GPS simulator's output signal strength. The $0-\mathrm{dB}$ setting, with a $30-\mathrm{dB}$ attenuator connected between the simulator and scintillation monitor, corresponds to the L1 C/A code signal level expected out of a 20 -dB preamplified antenna ( $\sim 140 \mathrm{dBW})$. Following an initial marker pulse at the $10-\mathrm{dB}$ setting and a 10-s idling period at $5 \mathrm{~dB}$, the linear frequency sweep (chirp) begins $20 \mathrm{~s}$ into the run. The chirp goes from 0 $\mathrm{Hz}$ to $25 \mathrm{~Hz}$ within $100 \mathrm{~s}$, while maintaining the magnitude of the signal strength fluctuation at $2 \mathrm{~dB}$ (peak).

The lower panel of Fig. 4 shows the scintillation monitor response to the chirp test. Power, on the vertical axis, is expressed in terms of arbitrary units of $\mathrm{WBP}_{k}$ in $\mathrm{dB}$ [i.e., $\left.10 \log _{10}\left(\mathrm{WBP}_{k}\right)\right]$. For reference, measured noise channel power, proportional to $N_{0} T_{c}$, usually falls around $20 \mathrm{~dB}$ on this scale. Clearly, the response of the scintillation monitor being tested falls off with increasing frequency. Fig. 5 shows the amplitude of the estimated transfer function between the input signal and the scintillation monitor's response. The -3 $\mathrm{dB}$ point lies at about $11 \mathrm{~Hz}$, while the response drops to -12 $\mathrm{dB}$ at $25 \mathrm{~Hz}$. 

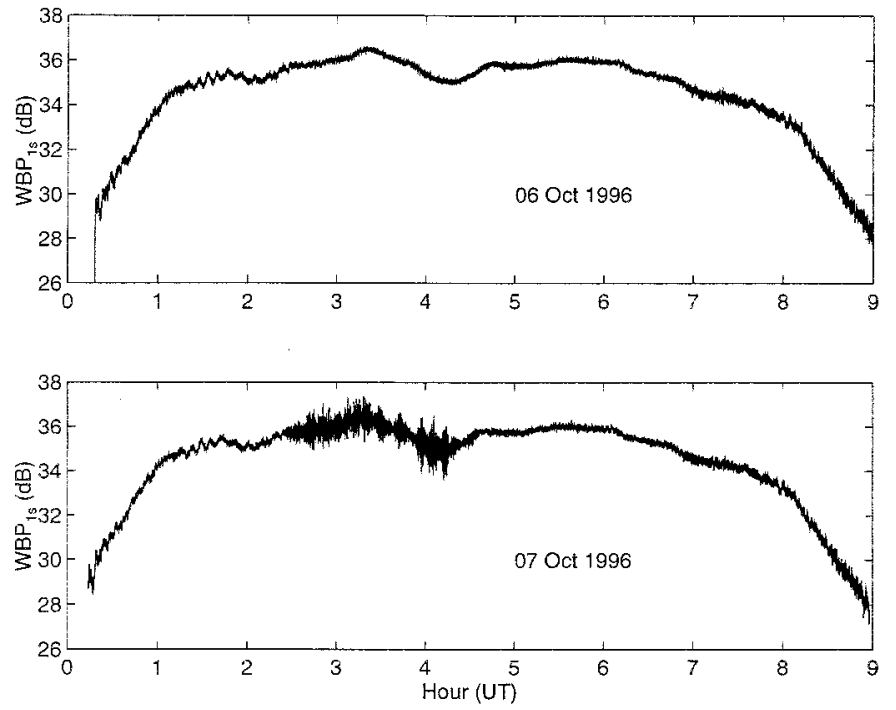

Fig. 6. Comparison of power measurements on successive days for the eastern receiver at Ancon, Peru. These data are $1 \mathrm{~s}$ averages of wide band power for PRN 14 measurements. Comparison of the two plots shows the repeatability of the background trend, including multipath, from day to day. Amplitude scintillations occurred between 0215 and 0415 UT on October 7 (2115-2315 LT on October 6).

Fig. 4 also clearly shows the measurement scatter in $\mathrm{WBP}_{k}$, particularly when the GPS simulator's output level is held constant in the segments from 0-10 s and 10-20 s into the run. Note that the biasing influence of the noise level on $\mathrm{WBP}_{k}$, as previously discussed and as explicitly shown in (7), is small for approximate quantitative work. For example, the change in mean amplitude between the 0 to $10 \mathrm{~s}$ and 10 to $20 \mathrm{~s}$ segments corresponds closely to the $-5 \mathrm{~dB}$ that the simulator setting changed. Similarly, during the early, low frequency part of the chirp the peak amplitude swing of $\mathrm{WBP}_{k}$ is approximately $2 \mathrm{~dB}$, which is the peak swing in the simulator's setting. Often, then, we shall study fluctuations in $\mathrm{WBP}_{k}$ (or $\mathrm{WBP}_{1 \mathrm{~s}}$ ) as representative of the scintillation fluctuation level. When calculating $S_{4}$, however, we remove the biasing effect of the noise level by subtracting low-pass filtered noise channel measurements.

\section{B. Field Testing-General Considerations}

So far, discussion has centered on two major sources of signal strength variations: amplitude scintillations and measurement scatter. Two other important sources of variation are satellite motion and multipath (usually also driven by satellite motion for stationary receivers). Multipath is especially critical to identify because it is possible for its fluctuation time scales to be comparable to those of scintillations.

Fig. 6 compares power measurements from PRN 14 at Ancon, Peru $\left(11.77^{\circ} \mathrm{S}, 77.15^{\circ} \mathrm{W}, 1.5^{\circ} \mathrm{dip}\right)$ for two successive nights. The second night, October 7, 1996, had scintillation activity. These plots show $\mathrm{WBP}_{1 \mathrm{~s}}, 1 \mathrm{~s}$ averages of wide band power, and therefore thickening of the traces due to measurement scatter is noticeably reduced from the bench-test examples.

The overall concave downward trend stems from satellite motion. Antenna patterns and varying satellite distance from the receiver combine to yield this background trend. The GPS satellites orbit with periods of 12 sidereal h (20 200-km altitude cir-
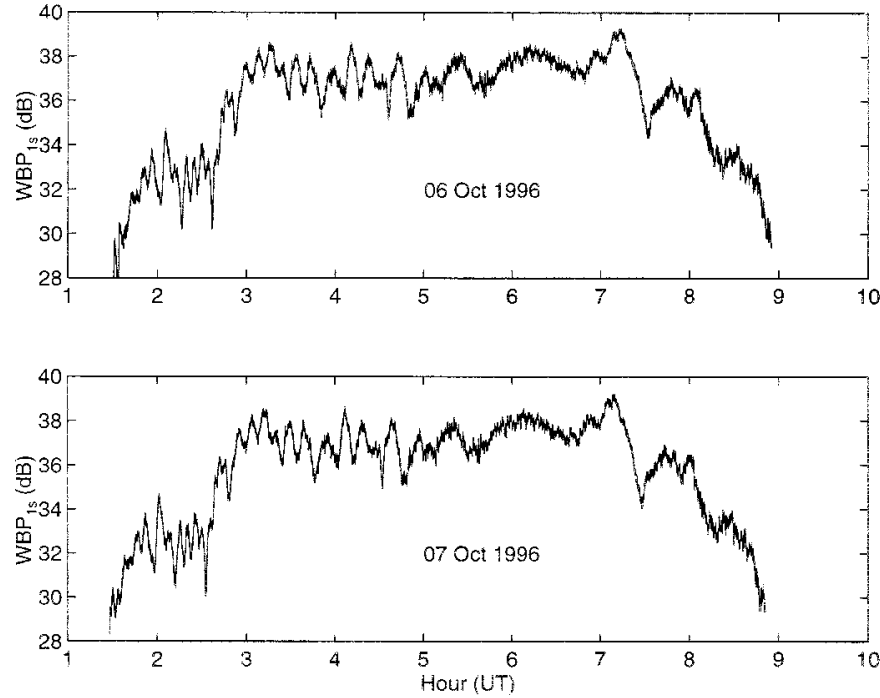

Fig. 7. Comparison of $1 \mathrm{~s}$ power averages on successive days for the Tucuman, Argentina receiver using PRN 14. The fine time scale (minutes to $10 \mathrm{~s}$ of minutes) variations that repeat from day to day are multipath effects.

cular orbits). Consequently, their ground tracks and the background patterns of signal strength variation repeat from night to night with the appropriate $\sim 4 \mathrm{~min} /$ day advance.

Multipath effects, too, repeat from night to night if the antenna's environment does not change. The quasi-sinusoidal ripples in signal strength around 0130 and 0800 UT on both nights are characteristic of multipath. More dramatically, Fig. 7 shows the multipath influence in PRN 14 data for the same two days from another scintillation monitor site in Tucuman, Argentina $\left(26.84^{\circ} \mathrm{S}, 65.22^{\circ} \mathrm{W},-25.0^{\circ}\right.$ dip). Even fine time scale $(\sim 1$ min) fluctuations repeat from night to night with a noticeable 4 min advance at the Tucuman site (despite multipath problems, the Tucuman station did observe scintillation on other nights and we have since improved the installation). Furthermore, at yet another scintillation monitor installation, we have observed patterns of "high frequency" (up to $10 \mathrm{~Hz}$ ) power fluctuations that repeat from night to night with the sidereal time shift.

Scintillations, in contrast, do not repeat from night to night. The example from Ancon (Fig. 6) displays a clear patch of scintillations between 0215 and 0415 UT on October 7, 1996 (the Tucuman station is about $2000 \mathrm{~km}$ SSE of Ancon and is south of the southern equatorial anomaly crest, so different scintillation patterns occur). Also, the time scales of amplitude scintillations are often shorter than those of multipath. The difference in time scales is clearly visible in the Ancon data of October 7, 1996. Nevertheless, such clear differentiation of time scales is not always evident.

For an order of magnitude calculation, the dominant horizontal scale of weak signal strength fluctuations due to ionospheric irregularities at zenith may be estimated from the peak of the Fresnel filtering function [1]. The peak of the Fresnel filtering function occurs at a scale size of $\sqrt{2 \lambda z}$, where $\lambda$ is the wavelength of the radio signal, and $z$ is the altitude of the irregularities. For the GPS L1 signal $(\lambda=0.19 \mathrm{~m})$ and $F$-peak irregularities $(z \approx 350 \mathrm{~km})$, the estimated horizontal scale size of signal strength fluctuations is $360 \mathrm{~m}$. Since the typical drift of the nighttime equatorial ionosphere is of the order of $100 \mathrm{~m} / \mathrm{s}$ 


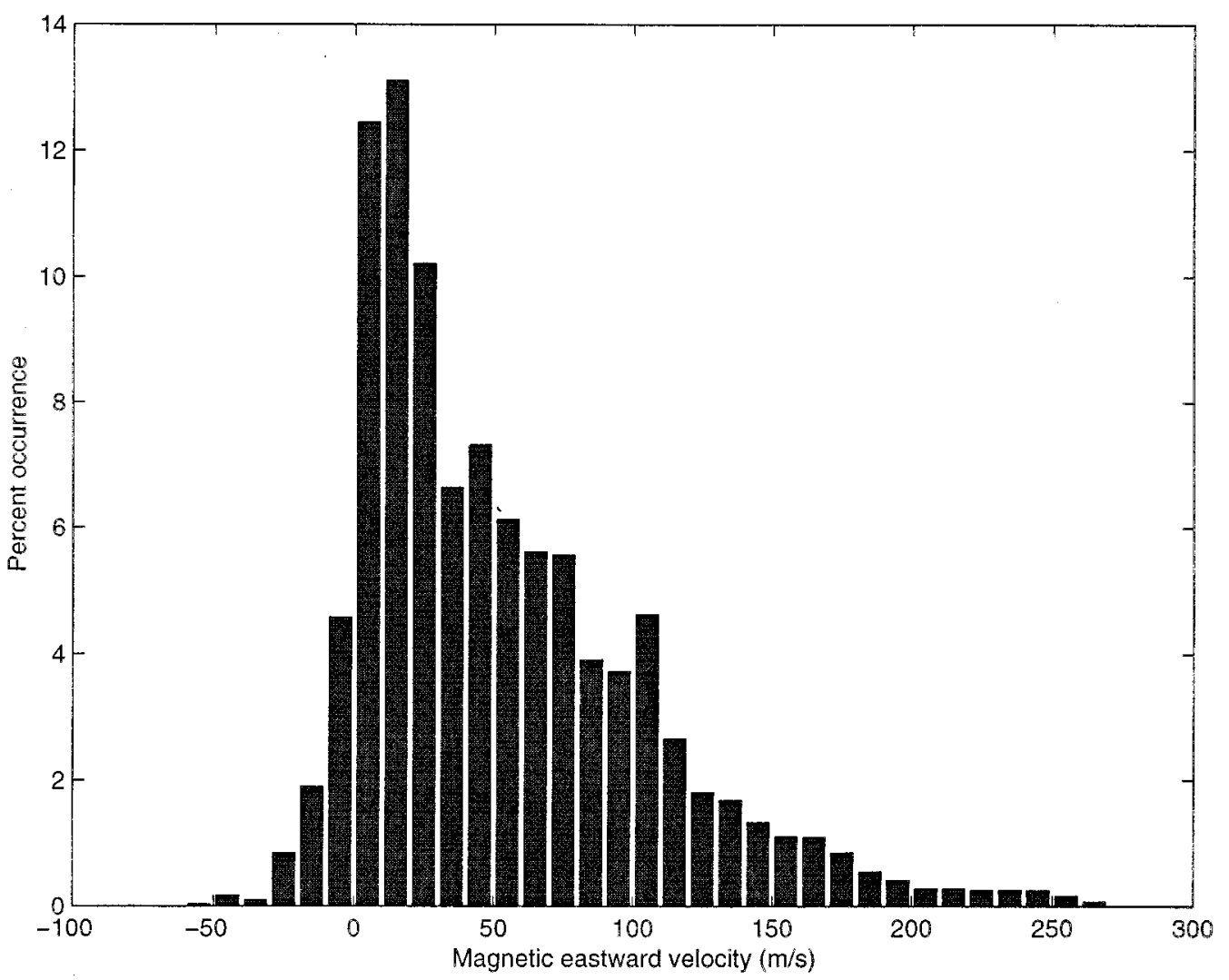

Fig. 8. Histogram of eastward velocities of the 350-km altitude ionospheric puncture point as seen from Ancon over a $24 \mathrm{~h}$ period on October 16, 1996. Positive velocities indicate motion from the geomagnetic west to the east, the same direction as the electrodynamically driven ionospheric plasma drift in early evening. The statistics are similar for a $12 \mathrm{~h}$ period covering only the nighttime.

toward the geomagnetic east [13], a characteristic weak scintillation time scale, or "fading rate," at the equator is approximately $4 \mathrm{~s}$. Again, this fading rate is only an order of magnitude estimate, but it matches well with the quasiperiods that the eye detects in amplitude patterns. (Note that the typical fading rate is about ten times greater for high-latitude scintillations, since horizontal plasma convection is of the order of $1000 \mathrm{~m} / \mathrm{s}$ in the polar cap [13]).

In reality, scintillation time scales vary due to variations in the ionospheric drift speed and changing distances to the irregularities. If the drift speed decreases or the irregularities are farther away, the fading rate is reduced. Satellite motion also contributes to the fading rate. The above scintillation time scale estimate assumed no net motion of the satellite relative to the receiver, or at least no motion of the satellite in the direction of ionospheric drift. Actually, the GPS satellites' ionospheric puncture points (i.e., their line of sight intersections with $F$-peak altitudes) have eastward components of motion ranging from $-50 \mathrm{~m} / \mathrm{s}$ to $200 \mathrm{~m} / \mathrm{s}$ and up (Fig. 8). If the puncture point moves with an eastward velocity comparable to the drift of the field-aligned irregularities, the fading rate decreases [14].

Thus, one situation in which discrimination between multipath and amplitude scintillation effects on signal strength becomes difficult is when velocity matching occurs. A severe enough reduction in fading rate makes it difficult to distinguish between the two effects. We have observed scintillations with fluctuation time scales of up to tens of seconds due to velocity matching. In a moderate-to-high multipath environment, the multipath fluctuations in signal strength are likely to have comparable time scales.

Fortunately, conditions are not normally so extreme. Commonly, equatorial scintillations have time scales of several seconds and, in an environment with relatively low multipath, most multipath remains on time scales of $100 \mathrm{~s}$ or greater. Under these conditions, scintillations can be separated from multipath by high-pass filtering. After all, researchers commonly detrend scintillation records to minimize the effects of satellite motion. Nevertheless, the possibility of confusing scintillations and multipath means that minimizing multipath is essential in any installation of the scintillation monitor.

The term "multipath" broadly covers any situation where direct and reflected signals reach the GPS antenna and interfere. Many authors are accustomed to thinking of GPS multipath as strictly a low-elevation effect, to be mitigated by elevation angle cutoffs and choke-ring antennas. Nevertheless, it is important to remember that reflections can occur at high elevation angles also. For example, nearby structures such as antennas and buildings can extend high into the field of view and reflect signals. One particularly subtle form of multipath occurs with raised antenna installations.

The preamplified antennas that we typically use with the Cornell scintillation monitor in the field are magnetically mounted L1 patch antennas with plastic housings. We place the antennas on small steel ground planes that we install at the experiment site. In the early campaigns, siting requirements sometimes forced us to raise these ground plane assemblies 


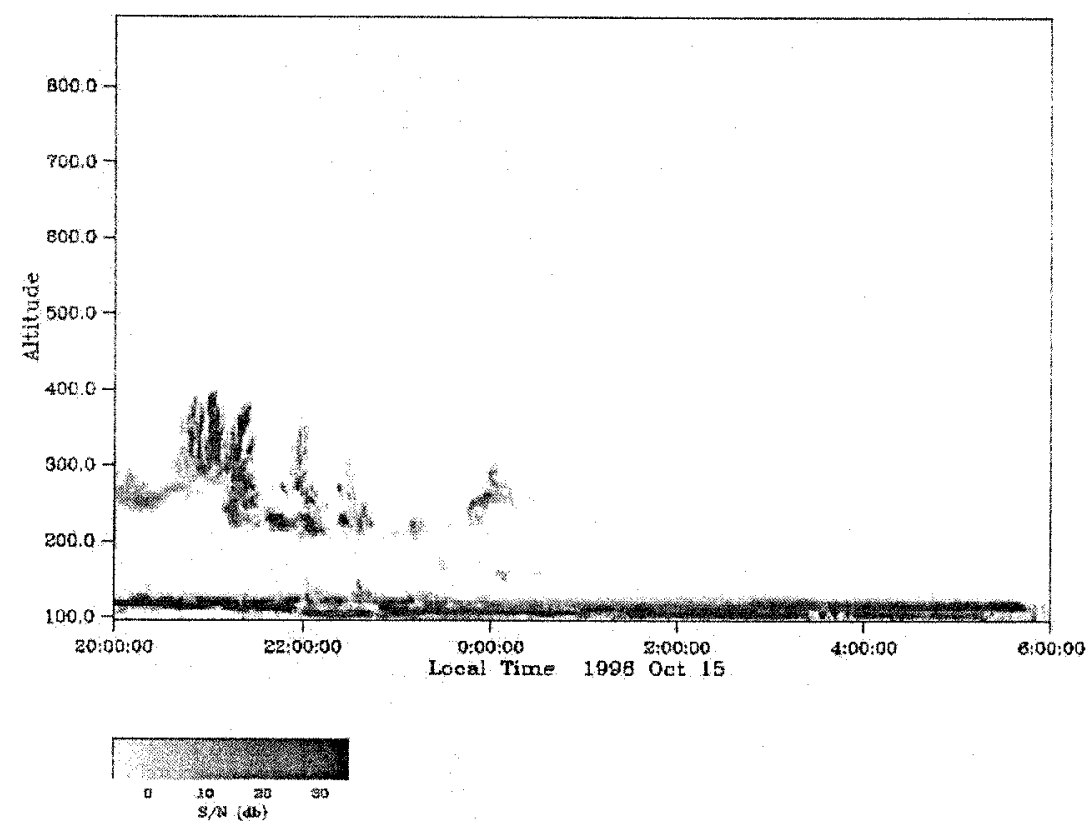

Fig. 9. Intensity of received echoes from ionospheric turbulence plotted as a function of altitude and time for the Jicamarca radar near Ancon and the dip equator. The plot traces equatorial spread F irregularities with scale sizes equal to half the 50-MHz radar's wavelength, approximately $3 \mathrm{~m}$. (Courtesy D. Hysell, Clemson University.)

above the ground or a roof (by setting the assembly on concrete blocks or a wooden frame, for example). We observed that raised installations tended to have more, and more rapid, multipath fluctuations (i.e., signal strength fluctuations that repeat from day to day with the appropriate sidereal advance) at higher elevation angles than sites where the ground plane lay directly on the ground or roof. Furthermore, remounting the antennas on larger steel ground planes or directly on the ground reduced the severity of the fluctuations at high elevation angles.

The likely culprit was interference of the direct signal with the reflected signal from the ground below the small ground plane. The situation is similar to the development of a large number of sidelobes in the pattern of an antenna raised above a perfectly conducting, infinite ground plane. In the case of the scintillation monitor installations, though, simple image theory does not strictly apply because of the presence of the small ground plane immediately below the antenna. Rather, the reflected signal diffracts around the edge of the small ground plane and reaches the patch antenna. In some general sense, the amplitude fluctuations caused by the interference of the direct and reflected signal in this case could possibly be termed "antenna pattern effects." We prefer, however, to classify all installation-specific radio wave interference effects that repeat from day to day, with the appropriate sidereal advance, as "multipath."

The point of this extended discussion is to provide a reminder that conventional wisdom on multipath does not necessarily apply when it comes to monitoring amplitude scintillations. We do not assert that all multipath effects observed in amplitude patterns are necessarily deleterious to normal GPS applications. Rather, we emphasize that GPS amplitude measurements are sensitive to interference between direct and reflected signals at all elevations. Therefore, common schemes where the GPS antenna is mounted on a pole or tripod may not be suitable for scintillation measurements. In general, the best guide to assessing the multipath environment of a particular installation is to compare amplitude traces from night to night (another technique, a technique that should also work for multipath from temporary changes in the antenna environment, is to monitor the code-carrier divergence [5]).

\section{Field Testing-Sample Results}

As important as it is to monitor and minimize multipath, the primary purpose of the Cornell scintillation monitor is to study amplitude scintillations. So far, this paper has identified scintillations by comparing the day to day repetition of signal strength patterns. Now we shall compare scintillation monitor measurements with measurements from other sensors: coherent scatter radar and a second scintillation monitor. These comparisons provide a brief, qualitative assessment of scintillation monitor research potential and additional confirmation of its proper functioning. Quantitative studies, such as the comparison of GPS total electron content fluctuations and amplitude scintillation observations [15], and systematic irregularity drift measurements by cross-correlation techniques [16], are addressed elsewhere.

Fig. 9 shows the range-time-intensity (RTI) $50-\mathrm{MHz}$ radar plot from Jicamarca, Peru for the night of October 15-16, 1996 (local time [LT]). Jicamarca is about $30 \mathrm{~km}$ southeast of Ancon. The radar returns trace coherent echoes from the 3-m scale ESF irregularities that drift or develop overhead [17]. The plot shows several "plumes" of ESF activity of relatively limited height extent. The 3-m scale irregularities form within regions of upwelling depletions in plasma density that extend north and south 


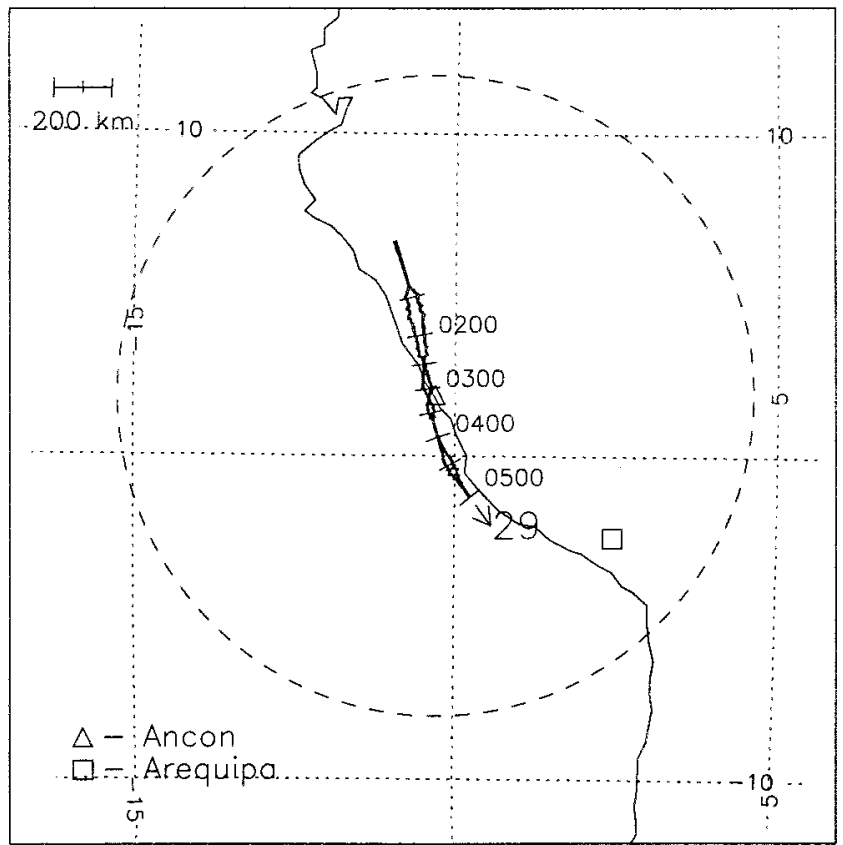

Fig. 10. Map of scintillation activity from the PRN 29 link at Ancon on October 6, 1996. The trail represents the 300-km altitude (mean $\mathrm{hmF} 2$ on this night) ionospheric puncture point of the satellite-to-receiver line of sight. Overlaid coordinates are geomagnetic (in degrees), and the dashed circle represents the $10^{\circ}$ elevation limit at $300-\mathrm{km}$ altitude. Labeled times are UT and a time tick marks the trail every half hour. The width of the trail is proportional to the scintillation index $S_{4}$. For reference, the width of a time tick corresponds to $S_{4}=0.4$.

along magnetic field lines, often as far as the equatorial anomaly regions (about $\pm 15^{\circ}$ dip latitude). The ESF depletions contain plasma density irregularities over several decades of scale size [13]. Once formed, the depletions and associated irregularities drift with the background plasma. The drift of the depletions is perpendicular to the magnetic field lines and toward the east in the early nighttime.

For comparison with the radar data, Fig. 10 shows the track of the 300-km altitude ionospheric puncture point of PRN 29's line of sight to Ancon on October 16, 1996 (UT). The diagram indicates the scintillation index $\left(S_{4}\right)$ at each point along the track by the width of the trail. Since PRN 29 moves roughly parallel to the field lines over Ancon and Jicamarca, the scintillation pattern (responding to irregularity scale sizes of $100-1000 \mathrm{~m}$ ) should show some degree of correspondence with the ESF activity observed by the radar due to the field-aligned nature of the irregularities (one limitation to good correspondence, for example, is that scale size governs the ability of irregularity-related perpendicular electric fields to map along the magnetic field lines; larger scale electric fields map more effectively [13]).

Some correspondence indeed exists. For example, the extended period of scintillations from 0130-0230 UT matches up well with the widest plume seen by the radar (2030-2130 LT; $\mathrm{LT}+5=\mathrm{UT}$ ). Nevertheless, we do not expect the correspondence to be so good at all times. After all, the measurements by the two techniques relate to scale sizes two orders of magnitude apart. Furthermore, the measurements are separated in magnetic latitude and slant path considerations also come into play for the GPS measurements.

As an example of noncorrespondence, from 0400-0500 UT, the radar echoes are faint but scintillation strength continues to remain at approximately the same level as during earlier events. Such behavior is consistent with previous observations of VHF and UHF scintillations with concurrent $50-\mathrm{MHz}$ radar measurements [18], [19]. A plausible scenario is that the plume has become a "fossil" or "dead" plume, and the smaller (3-m) scale irregularities have decayed away by diffusion.

Nevertheless, the coupling of GPS scintillation observations with radar observations on a systematic basis would provide for two potentially productive lines of inquiry. First, GPS observations provide information on irregularities from several lines of sight, covering a wide geographic area. Although coverage is admittedly sparse, GPS scintillation measurements can yield insight into the behavior of ESF structures, as traced by medium $(100-1000 \mathrm{~m})$ scale irregularities, to the east and west of the radar beam by 100s of km. Second, comparison of GPS scintillations and coherent scatter radar occasionally offers coincident means to examine the growth or decay of plasma structures two decades apart in scale.

The other example of coincident measurements comes from spaced receiver studies. At the Ancon site, during the October 1996 experimental campaign, we operated two scintillation monitors with their antennas spaced 100-m apart in the magnetic east-west direction. Since the scintillation monitor design uses GPS signal timing to synchronize data collection between nearby receivers, we were able to perform correlation studies. For example, Fig. 11 illustrates spaced receiver measurements of amplitude scintillations on PRN 14 for the night of October, 15-16, 1996. The patterns at both stations indicate a high degree of correlation and the fluctuation pattern appears at the western receiver about one second before repeating at the eastern receiver. This 1-s lag implies a west to east drift of the irregularities of the order of $100 \mathrm{~m} / \mathrm{s}$, relative to the motion of the GPS line of sight, consistent with typical early nighttime $F$-region drifts at the equator [13].

Clearly, though, some research must be done to provide accurate drift information from spaced-receiver GPS studies. First of all, the processing of scintillation-derived drifts has complications even in the simpler case of measurements from geostationary satellites [20]. In the case of GPS measurements, the motion of the line of sight through the ionosphere usually falls into a regime where neither standard assumption, "fixed ionosphere" (i.e., rapid satellite motion) or fixed satellite, readily applies. Also, any scintillation-based drift measurement does not provide information on the drifts prior to the formation of irregularities. Nevertheless, the Fig. 11 results demonstrate that crosscorrelation studies with spaced scintillation monitors are technically feasible and, despite complications, preliminary studies indicate that the use of spaced scintillation monitors to calculate irregularity drift is a promising technique [16]. The advantages of the GPS scintillation monitor in making drift measurements are, of course, that it is compact and relatively inexpensive and therefore can provide measurements in regions that are not well covered by other ionospheric instrumentation. 

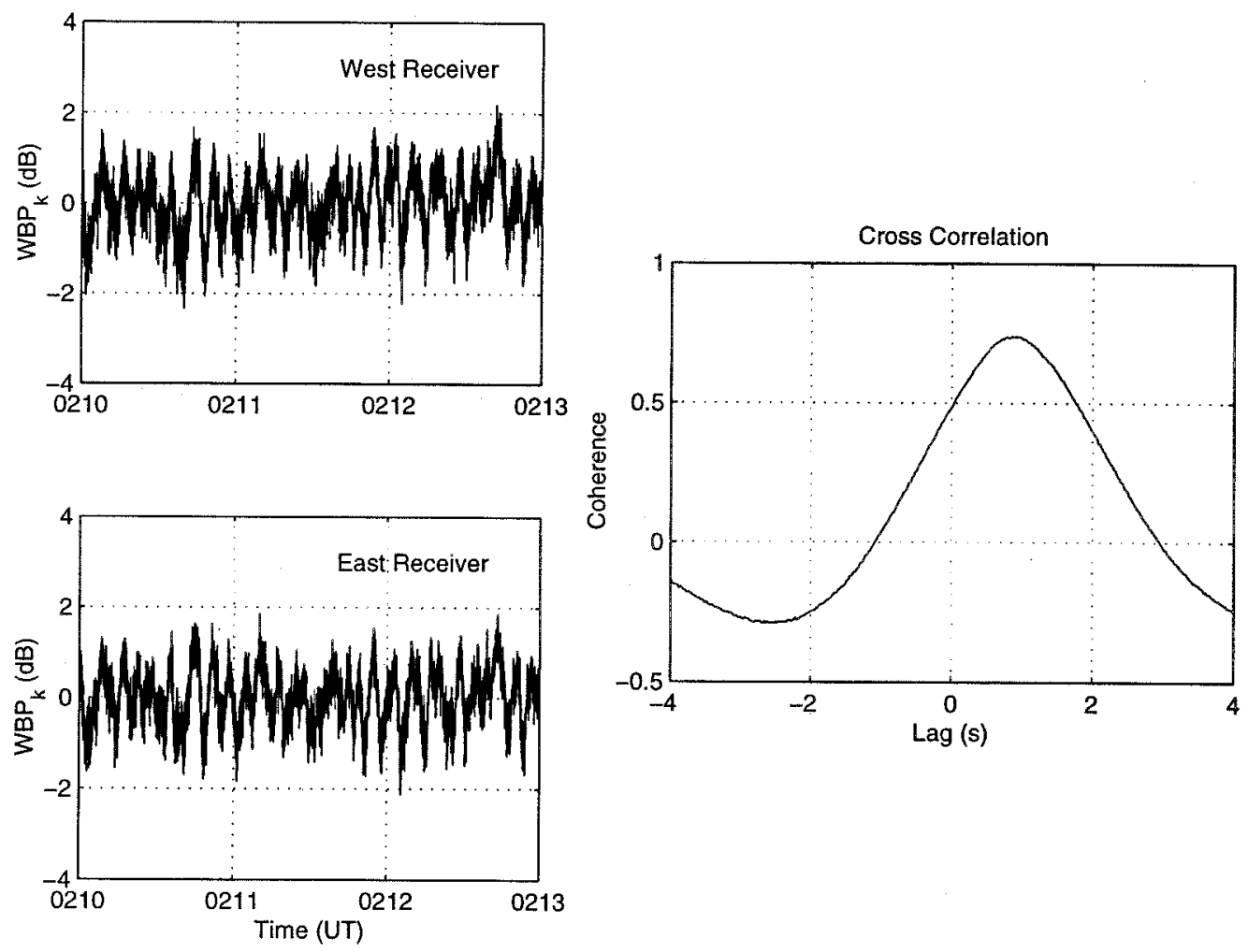

Fig. 11. Example of spaced receiver measurements. These data are from Ancon, Peru for PRN 14 on October 16, 1996. The left hand side shows the comparison of detrended power data between the western and eastern receivers. The right hand side plots the cross-correlation between the detrended power measurements.

\section{CONCLUding REMARKS}

We have demonstrated the successful modification of a commercial GPS receiver development system to monitor variations in signal strength at a rate of 50 samples/s. The Cornell scintillation monitor can record scintillations from several GPS lines of sight simultaneously and permits correlation studies with multiple receivers. Additionally, observations of signal strength fluctuations from day to day may provide insight into the multipath environment of a stationary antenna installation.

The scintillation monitor has been operated from many sites in South America (Ancon, Peru; Tucuman, Argentina; and several sites in Brazil), as well as in Africa, Alaska, Ascension Island, and Svalbard. Measurements continue with collaborators in South America and on a campaign basis with researchers from Cornell University or the Air Force Research Laboratory. Cornell researchers have also recently modified the receiver tracking loops to improve the receiver's ability to monitor phase scintillations. Correlative measurements are being conducted with GPS TEC measurements, airglow imaging, and ionosondes. Results from these investigations are being used to better characterize equatorial L-band scintillations and their potential effects on the operation of GPS receivers.

The Cornell scintillation monitor is an inexpensive and effective approach to monitoring and investigating L-band ionospheric scintillations. Several institutes and universities worldwide are now operating the monitor, which requires only a personal computer, the development system card in a 16-bit PC slot, and the executable code to acquire and log the amplitude scintillation data. During the current peak of solar activity, we antic- ipate investigating scintillation activity during its most intense disturbance of GPS signals.

\section{ACKNOWLEDGMENT}

The authors would like to thank R. Ezquer, J. Gary, and C. Martinis for providing support and operations at the Tucuman, Argentina site, and J. Espinoza and M. Ishitsuka for providing support at the Ancon, Peru site.

\section{REFERENCES}

[1] K. C. Yeh and C. H. Liu, "Radio wave scintillations in the ionosphere," Proc. IEEE, vol. 70, pp. 324-360, 1982.

[2] J. Aarons, "Global morphology of ionospheric scintillations," Proc. IEEE, vol. 70, pp. 360-378, 1982.

[3] S. Basu, E. MacKenzie, and S. Basu, "Ionospheric constraints on VHF/UHF communications links during solar maximum and minimum periods," Radio Sci., vol. 23, pp. 363-378, 1988.

[4] "GEC plessey semiconductors," in Global Positioning: Products Handbook: Publ. HB4305, GEC Plessey Semiconductors, Aug. 1996.

[5] A. J. Van Dierendonck, J. Klobuchar, and Q. Hua, "Ionospheric scintillation monitoring using commercial single frequency C/A code receivers," in Proc. ION GPS-93, Arlington, VA, Sept. 1993, pp. 1333-1342.

[6] J. A. Klobuchar, S. Basu, Q. Hua, and A. J. Van Dierendonck, "A versatile amplitude and phase scintillation monitor using a GPS commercial single frequency C/A code receiver," in Proc. Int. Beacon Satellite Symp.. Aberystwyth, U.K., July 11-15, 1994, pp. 250-253.

[7] B. H. Briggs, G. J. Phillips, and D. H. Shinn, "The analysis of observations on spaced receivers of the fading of radio signals," Proc. Phys. Soc. London B, vol. 63, pp. 106-121, 1950.

[8] J. J. Spilker, "GPS signal structure and theoretical performance," in Global Positioning System: Theory and Applications, B. W. Parkinson and J. J. Spilker, Eds. Washington, DC: AIAA, 1996, vol. I, pp. $57-119$.

[9] A. J. Van Dierendonck, "GPS receivers," in Global Positioning System: Theory and Applications, B. W. Parkinson and J. J. Spilker, Eds. Washington, DC: AIAA, 1996, vol. I, pp. 329-407. 
[10] S. Hinedi and J. I. Statman, "Digital accumulators in phase and frequency tracking loops," IEEE Trans. Aerosp. Electron. Syst., vol. AES-26, pp. 169-180, 1980.

[11] C. R. Cahn, D. K. Leimer, C. L. Marsh, F. J. Huntowski, and G. D. LaRue, "Software implementation of a PN spread spectrum receiver to accommodate dynamics," IEEE Trans. Commun., vol. COM-25, pp. 832-840, 1977.

[12] A. Papoulis, Probability, Random Variables, and Stochastic Processes, 3rd ed. New York: McGraw-Hill, 1991.

[13] M. C. Kelley, The Earth's Ionosphere: Plasma Physics and Electrodynamics. San Diego, CA: Academic, 1989.

[14] P. M. Kintner, H. Kil, T. L. Beach, and E. R. de Paula, "Fading time scales associated with GPS signals and potential consequences," Radio Sci., to be published.

[15] T. L. Beach and P. M. Kintner, "Simultaneous global positioning system observations of equatorial scintillations and total electron content fluctuations," J. Geophys. Res., vol. 104, pp. 22 553-22 565, 1999.

[16] H. Kil, P. M. Kintner, E. R. de Paula, and I. J. Kantor, "Global positioning system measurements of the ionospheric zonal apparent velocity at Cachoeira Paulista in Brazil,” J. Geophys. Res., vol. 105, pp. 5317-5327, 2000.

[17] R. F. Woodman and C. La Hoz, "Radar observations of F region equatorial irregularities," J. Geophys. Res., vol. 81, pp. 5447-5466, 1976.

[18] S. Basu, J. P. McClure, S. Basu, W. B. Hanson, and J. Aarons, "Coordinated study of equatorial scintillations and in situ and radar observations of nighttime F region irregularities," J. Geophys. Res., vol. 85, pp. 5119-5130, 1980.

[19] S. Basu, S. Basu, J. LaBelle, E. Kudeki, B. G. Fejer, M. C. Kelley, H. E. Whitney, and A. Bushby, "Gigahertz scintillations and spaced receiver drift measurements during project Condor equatorial $\mathrm{F}$ region rocket campaign in Peru," J. Geophys. Res., vol. 91, pp. 5526-5538, 1986.

[20] A. W. Wernik, C. H. Liu, and K. C. Yeh, "Modeling of spaced-receiver scintillation measurements," Radio Sci., vol. 18, pp. 743-764, 1983.

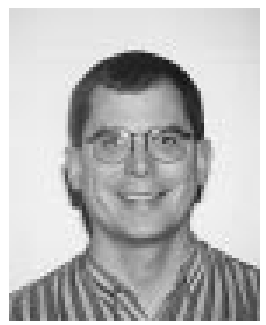

Theodore L. Beach (S'94-M'97) received the B.S.E.E. and M.S.E.E. degrees from Ohio University, Athens, in 1987 and 1988, respectively, and the $\mathrm{Ph} . \mathrm{D}$. degree in electrical engineering from Cornell University, Ithaca, NY, in 1998, where his dissertation research concentrated on monitoring ionospheric disturbances (traveling ionospheric disturbances and equatorial scintillations) with GPS receivers.

$\mathrm{He}$ is currently an Electronics Engineer with the Space Vehicles Directorate, Air Force Research Laboratory, Hanscom Air Force Base, MA. While pursuing the M.S. degree, he worked for the Avionics Engineering Center, Ohio University, on GPS-related issues. He subsequently trained personnel for the United States Navy and investigated an opportunity for company ownership. He also holds registration as a Professional Engineer in Ohio.

Dr. Beach is a member of Eta Kappa Nu, Phi Kappa Phi, Tau Beta Pi, and the American Geophysical Union.

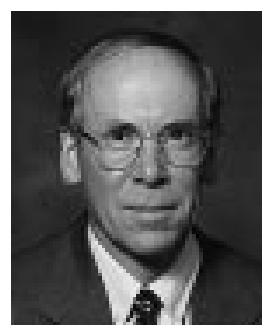

Paul M. Kintner (SM'82) received the Ph.D. degree from the Physics Department, University of Minnesota, Minneapolis, in 1974

$\mathrm{He}$ is currenty a Professor of electrical engineering, Cornell University, Ithaca, NY. He was a Research Associate with the University of Iowa, Iowa City, and Cornell University before joining the faculty of Cornell University in 1981. His research has made seminal contributions to the understanding of the near-space environment and to the development of instrumentation for both in situ sensing and remote sensing. His most well known instrument is the plasma wave interferometer for measuring the phase velocity and wavelength of space plasma waves and the scale size of discrete plasma structures in the ionosphere. Using this and similar instruments, he is credited with the discovery of electrostatic ion cyclotron waves, double layers, and lower hybrid solitary structures in space. His work is frequently cited for understanding the ionosphere as a mass source for the magnetosphere. Recently, he developed a program using the global positioning system (GPS) to remotely sense the ionosphere. GPS receivers designed in this program are used to monitor ionospheric scintillations and ionospheric drifts. In an additional development, GPS receivers designed in this program are being used for time synchronization on spacecraft. He was a Member of the NASA Working Group on the Suborbital Program.

Prof. Kintner has served as a Co-Convener for a variety of conferences and participated in innumerable NASA review panels. He is a member of the American Geophysical Union and has developed and teaches courses on GPS (theory and design and space science and engineering) at Cornell University. 